\title{
MODULACIÓN DE VECTOR ESPACIAL PARA INVERSORES TRIFÁSICOS CON CUATRO RAMAS DE INTERRUPTORES
}

\section{SPACE VECTOR MODULATION OF THREE PHASE FOUR LEG INVERTER}

\author{
PhD. Omar Pinzón Ardila.
}

Universidad Pontificia Bolivariana, Seccional Bucaramanga. Facultad de Ingeniería Electrónica, Grupo de Investigación en Control Industrial. Km. 7, vía Piedecuesta, Bucaramanga, Santander, Colombia. Tel: (+577) 6796220 .

E-mail: omar.pinzon@upb.edu.co

\begin{abstract}
Resumen: Este artículo analiza una modulación de vector espacial en tres dimensiones de un inversor con cuatro ramas de interruptores y determina los valores máximos y mínimos de tensión homopolar que puede suministrar el inversor en régimen permanente. Se encuentra que en el cálculo de los modos activos existe una independencia con respecto a la tensión homopolar dada, por tal razón se puede calcular en forma convencional la modulación de ancho de pulso y sólo es necesario ajustar el cálculo de los modos inactivos y el tiempo de conmutación de la cuarta rama de interruptores para inyectar tensión homopolar en el sistema. Además, se propone un nuevo método de cálculo de tiempos inactivos y de la cuarta rama de interruptores. Por último, se valida el procedimiento empleado en simulación y se valida la metodología propuesta empleando un prototipo en laboratorio.
\end{abstract}

Palabras clave: Convertidores fuente de tensión, inversores, modulación de vector espacial, Filtros Activos, sistemas trifásicos con hilo neutro.

\begin{abstract}
This paper discusses a space vector modulation of a three phase four leg inverter and determines the maximum and minimum values of zero sequence voltage that can generate an inverter in state stable. Independence between active modes time and zero sequence voltage is founded therefore modulation pulse width modulation can be calculated using a classic three-phase three-leg inverter therefore it is only necessary to calculate the inactive-mode time and switching time of fourth leg to inject zero sequence component into the system. In addition, a new method is proposed to calculate switching time of inactive mode and four-leg. Finally, the procedure is validated in simulation and the method proposed is validated using a laboratory prototype.
\end{abstract}

Keywords: Voltage-source converters, three phase inverters, space vector modulation, active power filter, four leg inverter, zero sequence components.

\section{INTRODUCCIÓN}

La utilización de inversores trifásicos con hilo neutro ha ido en ascenso debido a la implementación de filtros activos en cargas localizadas en sistemas de distribución con hilo neutro, por tal motivo, es necesario el estudio de inversores con hilo neutro con el propósito de controlar un nuevo grado de libertad que proporciona la tensión o corriente homopolar. Estudios (Zhang, 2002; Shen y Lehn, 2002; Xiaoping et al. 2008; Dai et al. 2006) muestran la 
modulación de ancho de pulso utilizando la técnica de vector espacial como espacios geométricos en tres dimensiones con un coste computacional elevado y un cambio total de los algoritmos existentes. Por tal razón se intenta simplificar los cálculos en inversores con ocho interruptores y realizar las mínimas modificaciones en los algoritmos existentes.

El presente trabajo estudia los valores máximos y mínimos de tensión homopolar que puede suministrar un inversor trifásico con ocho interruptores y son aplicados a la técnica de vector espacial ampliada en un grado de libertad. Este análisis es utilizado y extendido para determinar los límites máximos y mínimos de tensión homopolar en régimen permanente. Además se propone un algoritmo que hace posible la implementación de dicho inversor ampliando los algoritmos existentes de inversores trifásicos sin hilo neutro.

\section{TÉCNICA DE VECTOR ESPACIAL APLICADA A INVERSORES CON OCHO INTERRUPTORES}

En esta sección se estudia la técnica de Vector Espacial aplicada a inversores trifásicos con ocho interruptores, para esto se apoya en la técnica de Vector Espacial convencional en el plano $d q$ e incorpora la tensión homopolar en las expresiones.

En la Figura 1 muestra el esquema simplificado de un inversor trifásico con ocho interruptores ideales de potencia. En este inversor existen limitaciones de operación en cuanto a la situación de los interruptores (Holtz, 1994; Pinzon, 2015; Srikanthan and Mishra, 2010). Una primera limitación indica que no pueden estar cerrados al mismo tiempo dos interruptores de una misma rama porque causaría un cortocircuito. La siguiente limitación es la imposibilidad de dejar abiertos los interruptores de una rama ya que la tensión de fase correspondiente en la carga no estaría determinada. Con las limitaciones anteriores y teniendo en cuenta que el estado de una rama es determinada con el conocimiento de la situación de un interruptor de la misma, aparecen 16 posibles combinaciones que son llamados modos del inversor. Estos modos se muestran en la Tabla 1 , la cual presenta los posibles estados de los interruptores $S_{1}, S_{2}, S_{3}$ y $S_{4}$ respectivamente. El número 1 en la columna $S_{1234}$ indica que el interruptor correspondiente se encuentra cerrado y su complemento denotado con una barra se encuentra abierto.

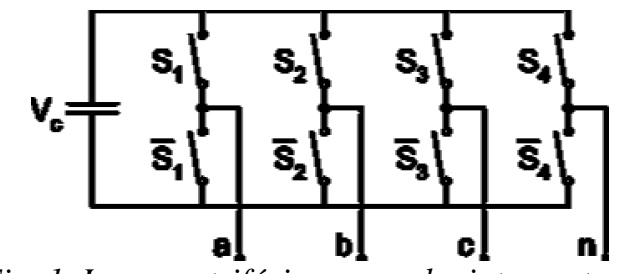

Fig. 1. Inversor trifásico con ocho interruptores.

Al aplicar al inversor de la Figura 1 cada uno de los modos se obtiene las tensiones de fase de las ramas $a, b$ y $c$ con respecto al punto $n$. La Tabla 1 reúne los resultados para cada modo y se incluye la tensión en ejes $d$, q y 0 del inversor utilizando la transformada de Park (1), esta transformación mantiene invariante la expresión de la potencia y permite transformar las tensiones de fase $a, b$ y $c$ con respecto al punto en un sistema coordenado ortogonal $0 d q$. Además, esta transformada implícitamente selecciona un sistema de referencia estático con el eje $d$ en fase con la fase $a$ ( $V_{d}$ alcanza su máximo cuando lo hace $V_{a}$ ).

\section{Tabla 1: Tensiones de fase y del inversor con ocho}

\begin{tabular}{crrrrrrr}
\multicolumn{7}{c}{ interruptores. } \\
\hline Modo & $S_{1234}$ & $V_{a n}$ & $V_{b n}$ & $V_{c n}$ & $V_{0}$ & $V_{d}$ & $V_{q}$ \\
\hline \hline $0^{+}$ & 0000 & 0 & 0 & 0 & 0 & 0 & 0 \\
$1^{+}$ & 1000 & $V_{c}$ & 0 & 0 & $\frac{V_{c}}{\sqrt{3}}$ & $\sqrt{\frac{2}{3}} V_{c}$ & 0 \\
$2^{+}$ & 1100 & $V_{c}$ & $V_{c}$ & 0 & $\frac{2 V_{c}}{\sqrt{3}}$ & $\frac{V_{c}}{\sqrt{6}}$ & $\frac{V_{c}}{\sqrt{2}}$ \\
$3^{+}$ & 0100 & 0 & $V_{c}$ & 0 & $\frac{V_{c}}{\sqrt{3}}$ & $-\frac{V_{c}}{\sqrt{6}}$ & $\frac{V_{c}}{\sqrt{2}}$ \\
$4^{+}$ & 0110 & 0 & $V_{c}$ & $V_{c}$ & $\frac{2 V_{c}}{\sqrt{3}}$ & $-\sqrt{\frac{2}{3} V_{c}}$ & 0 \\
$5^{+}$ & 0010 & 0 & 0 & $V_{c}$ & $\frac{V_{c}}{\sqrt{3}}$ & $-\frac{V_{c}}{\sqrt{6}}$ & $-\frac{V_{c}}{\sqrt{2}}$ \\
$6^{+}$ & 1010 & $V_{c}$ & 0 & $V_{c}$ & $\frac{2 V_{c}}{\sqrt{3}}$ & $\frac{1 c}{\sqrt{6}}$ & $-\frac{V_{c}}{\sqrt{2}}$ \\
$7^{+}$ & 1110 & $V_{c}$ & $V_{c}$ & $V_{c}$ & $\sqrt{3} V_{c}$ & 0 & 0 \\
$0^{-}$ & 0001 & $-V_{c}$ & $-V_{c}$ & $-V_{c}$ & $-\sqrt{3} V_{c}$ & 0 & 0 \\
$1^{-}$ & 1001 & 0 & $-V_{c}$ & $-V_{c}$ & $-\frac{2 V_{c}}{\sqrt{3}}$ & $\sqrt{\frac{2}{3}} V_{c}$ & 0 \\
$2^{-}$ & 1101 & 0 & 0 & $-V_{c}$ & $-\frac{V_{c}}{\sqrt{3}}$ & $\frac{V_{c}}{\sqrt{6}}$ & $\frac{V_{c}}{\sqrt{2}}$ \\
$3^{-}$ & 0101 & $-V_{c}$ & 0 & $-V_{c}$ & $-\frac{2 V_{c}}{\sqrt{3}}$ & $-\frac{V_{c}}{\sqrt{6}}$ & $\frac{V_{c}}{\sqrt{2}}$ \\
$4^{-}$ & 0111 & $-V_{c}$ & 0 & 0 & $-\frac{V_{c}}{\sqrt{3}}$ & $-\sqrt{\frac{2}{3} V_{c}}$ & 0 \\
$5^{-}$ & 0011 & $-V_{c}$ & $-V_{c}$ & 0 & $-\frac{2 V_{c}}{\sqrt{3}}$ & $-\frac{V_{c}}{\sqrt{6}}$ & $-\frac{V_{c}}{\sqrt{2}}$ \\
$6^{-}$ & 1011 & 0 & $-V_{c}$ & 0 & $-\frac{V_{c}}{\sqrt{3}}$ & $\frac{V_{c}}{\sqrt{6}}$ & $-\frac{V_{c}}{\sqrt{2}}$ \\
$7^{-}$ & 1111 & 0 & 0 & 0 & 0 & 0 & 0 \\
\hline
\end{tabular}

Por otra parte, en los modos $0^{+}, 0^{-}, 7^{+}$y $7^{-}$la tensión aplicada por el inversor en ejes es cero, por tal razón estos modos son llamados modos inactivos, mientras que los modos del $1^{+/-}$al $6^{+/-}$ son llamados modos activos.

En la Figura 2 se muestran los modos representados en tres dimensiones donde sus ejes son directo, cuadratura y homopolar. La parte (a) representa los modos contenidos en una superficie envolvente, esta superficie permite determinar la región factible del vector de espacio requerido por el inversor. La parte (b) muestra los vectores de espacio que conforman los 16 modos del inversor trifásico. Para mayor claridad se indican en líneas discontinuas aquellos modos localizados atrás del punto de vista dado. 


$$
\left[\begin{array}{l}
V_{0} \\
V_{d} \\
V_{q}
\end{array}\right]=\sqrt{\frac{2}{3}}\left[\begin{array}{rrr}
\frac{1}{\sqrt{2}} & \frac{1}{\sqrt{2}} & \frac{1}{\sqrt{2}} \\
1 & -\frac{1}{2} & -\frac{1}{2} \\
0 & \sqrt{\frac{3}{2}} & -\sqrt{\frac{3}{2}}
\end{array}\right]\left[\begin{array}{l}
V_{a} \\
V_{b} \\
V_{c}
\end{array}\right]
$$

Por otra parte, la Figura 3 muestra la proyección de la Figura 2 sobre el plano $d q$ y a su derecha la cantidad de componente homopolar que puede suministrar dichos modos. El resultado en el plano $d q$ es la figura bien conocida de la modulación del vector espacial de un inversor sin hilo neutro. En esta proyección los modos de igual número pero diferente superíndices $(+/-)$ se encuentran contenidos en el mismo punto y las líneas continuas indican modos activos adyacentes. Además, se observa que la tensión homopolar suministrada por los modos depende linealmente de la tensión del condensador del inversor.
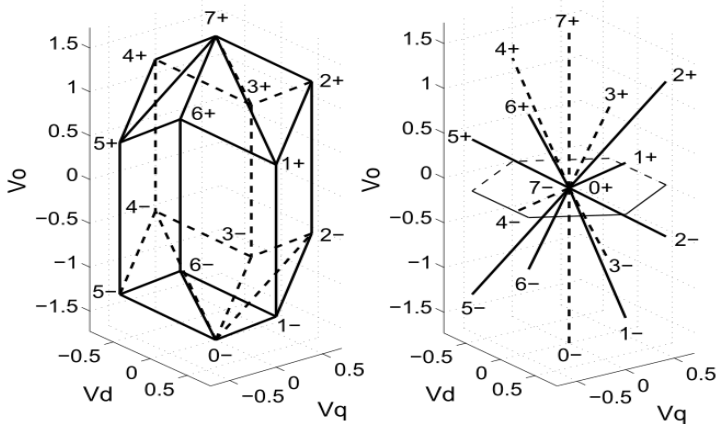

Fig. 2. Modos en ejes $0 d q$ del inversor trifásico con 8 interruptores. (a) Representación espacial del envolvente volumétrico que contiene los modos.

(b) Lugar geométrico de la tensión del inversor con componente homopolar.
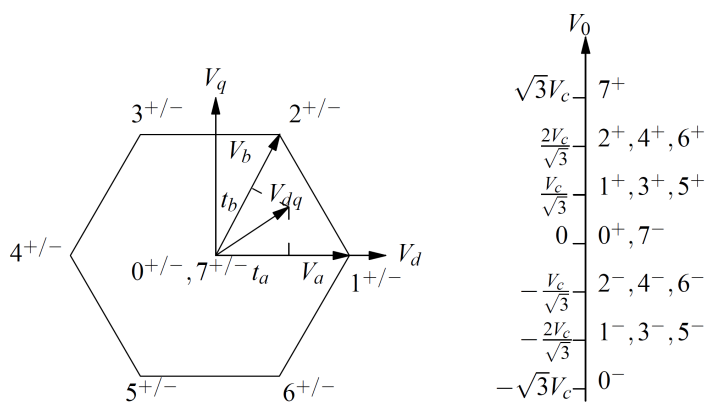

Fig. 3. Modos proyectados en el plano dq. Las líneas continuas indican los modos adyacentes. Los superíndices de los modos $+y-$ se proyectan sobre el mismo punto. (b) Componente homopolar suministrada por cada modo.
El objetivo final del inversor es suministrar una tensión en terminales del inversor, para esto es necesario conmutar el inversor entre varios modos adyacentes durante un periodo de conmutación (Holtz, 1994), de forma tal, que el valor medio de la tensión generada coincida con la onda deseada. Sí se define $a$ y $b$ como los modos activos que subyacen un sector dado y en el que se encuentra la tensión a ser suministrada por el inversor se puede obtener un vector de tensión del inversor $\mathbf{V}_{0 d q}$ (2) al aplicar durante un tiempo $t_{a}$ la tensión $\mathbf{V}_{0 d q}^{a}$ en el modo activo $a$ (modos 1,3 y 5) y durante un tiempo $t_{b}$ la tensión $\mathbf{V}_{0 d q}^{b}$ del modo activo $\mathrm{y}$ adyacente $b$ (modos 2, 4 y 6). De la misma forma se aplica para los modos inactivos. Al igual se debe cumplir siempre la restricción de tiempo total dada en (3).

$$
\begin{gathered}
\mathbf{V}_{0 d q}=t_{a} \mathbf{V}_{0 d q}^{a}+t_{b} \mathbf{V}_{0 d q}^{b}+t_{0} \mathbf{V}_{0 d q}^{0}+t_{7} \mathbf{V}_{0 d q}^{7} \\
1=t_{a}+t_{b}+t_{0}+t_{7}
\end{gathered}
$$

Donde $t_{x}$ ( $x$ indica cualquier modo $a, b, 0$ o 7 ) es el tiempo total, normalizado con respecto al periodo de conmutación, empleado por el modo $x$ $\left(t_{x}=t_{x}^{+}+t_{x}^{-}\right)$y $\mathbf{V}_{0 d q}^{x}$ el vector de tensión dado por la Tabla 1 .

Para obtener tensión del inversor es necesario calcular los tiempos de los modos activos e inactivos. Las siguientes secciones se muestra la forma de calcular dichos tiempos y como estos se seleccionan con la tensión homopolar.

\section{CÁLCULO DE LA TENSIÓN HOMOPOLAR Y TIEMPOS DE CONMUTACIÓN}

Como se ha descrito en la sección anterior, un inversor con ocho interruptores presenta 16 modos de conmutación, tal como lo muestra la Tabla 1 . Fundamentalmente, la Tabla 1 está dividida en dos partes. Los modos $0^{+}-7^{+}$que proporcionan la componente homopolar positiva y los modos $0^{-}$$7^{-}$que suministran la componente homopolar negativa.

Al reescribir la expresión (2) obtiene (4) donde se desagregan los tiempos empleados por cada uno de los modos implicados en suministran tensión homopolar positiva y negativa. 


$$
\begin{aligned}
\mathbf{V}_{0 d q}= & t_{a}^{+} \mathbf{V}_{0 d q}^{+a}+t_{b}^{+} \mathbf{V}_{0 d q}^{+b}+t_{0}^{+} \mathbf{V}_{0 d q}^{+0}+t_{7}^{+} \mathbf{V}_{0 d q}^{+7} \\
& +t_{a}^{-} \mathbf{V}_{0 d q}^{-a}+t_{b}^{-} \mathbf{V}_{0 d q}^{-b}+t_{0}^{-} \mathbf{V}_{0 d q}^{-0}+t_{7}^{-} \mathbf{V}_{0 d q}^{-7}
\end{aligned}
$$

Donde $+a$ indica un modo activo del inversor contenido en la mitad superior de la Tabla 1. - indica un modo activo del inversor contenido en la mitad inferior de la Tabla 1. $+b$ indica un modo activo adyacente a $+a$ y contenido en la mitad superior de la Tabla 1. $-b$ indica un modo activo adyacente a $-a$ y contenido en la mitad inferior de la Tabla 1. Los modos activo y adyacentes presentan una diferencia entre ellos de $60^{\circ}$ y están medidos en la proyección del plano $d q$.

Sin pérdida de generalidad, se estudia cómo obtener una tensión total del inversor contenida en el subespacio del primer sector espacial (modos 1 2 ) (Holtz, 1994; Zhang et al. 2012).

Al reemplazar los valores correspondientes de la Tabla 1 en (4) y normalizando con respecto a la tensión del condensador, aparecen tres expresiones en unitarias que son la tensión en eje directo $v_{d}$ (5), la tensión en eje en cuadratura $v_{q}$ (6) y la tensión homopolar que se muestra más adelante.

$$
\begin{gathered}
v_{d}=\sqrt{\frac{2}{3}} t_{a}+\frac{1}{\sqrt{6}} t_{b} \\
v_{q}=\frac{1}{\sqrt{2}} t_{b}
\end{gathered}
$$

Se observa en las expresiones de tensión $v_{d}$ y $v_{q}$ existe independencia con respecto a los modos no activos. Por tal razón, se puede determinar el tiempo total de cada modo activo sin emplear la tensión homopolar.

Al resolver el sistema dado por las (5) y (6) se obtiene los tiempos de los modos activos como se muestra en (7). Este resultado es igual al caso bien conocido de un del inversor con seis interruptores sin hilo neutro.

$$
\left[\begin{array}{l}
t_{a} \\
t_{b}
\end{array}\right]=\sqrt{2}\left[\begin{array}{cc}
\frac{\sqrt{3}}{2} & -\frac{1}{2} \\
0 & 1
\end{array}\right]\left[\begin{array}{l}
v_{d} \\
v_{q}
\end{array}\right]
$$

Siguiendo con el cálculo de la tensión homopolar, la tensión homopolar del inversor en (8) está dada como la suma de las tensiones homopolares suministradas por cada modo involucrado en la conmutación del inversor (4).

$$
\begin{gathered}
V_{0}=V_{0}^{a+} t_{+a}+V_{0}^{a-} t_{-a}+V_{0}^{b+} t_{+b}+V_{0}^{b-} t_{-b} \\
+V_{0}^{0+} t_{+0}+V_{0}^{0-} t_{-0}+V_{0}^{7+} t_{+7}+V_{0}^{7-} t_{-7}
\end{gathered}
$$

Sí al descomponer la tensión homopolar suministrada por cada modo como la tensión homopolar media suministrada por cada modo y un valor incremental de tensión como sigue:

$$
\begin{gathered}
V_{0}^{a+}=-\bar{V}+\Delta V \\
V_{0}^{a-}=-\bar{V}-\Delta V \\
V_{0}^{b+}=\bar{V}+\Delta V \\
V_{0}^{b-}=\bar{V}-\Delta V \\
V_{0}^{0+}=-3 \bar{V}+\Delta V \\
V_{0}^{0-}=-3 \bar{V}-\Delta V \\
V_{0}^{7+}=3 \bar{V}+\Delta V \\
V_{0}^{7-}=3 \bar{V}-\Delta V
\end{gathered}
$$

Donde $\bar{V}=\frac{V c}{2 \sqrt{3}}$ corresponde al valor medio que suministra un modo activo y $\Delta V=\frac{V c \sqrt{3}}{2}$ al incremento necesario para obtener el valor de tensión homopolar deseado.

Además, se definen los tiempos de la cuarta rama de interruptores $S_{4}$ como $t^{+}$y $t^{-}$tal y como se muestra en la Figura 4 y sus expresiones como sigue:

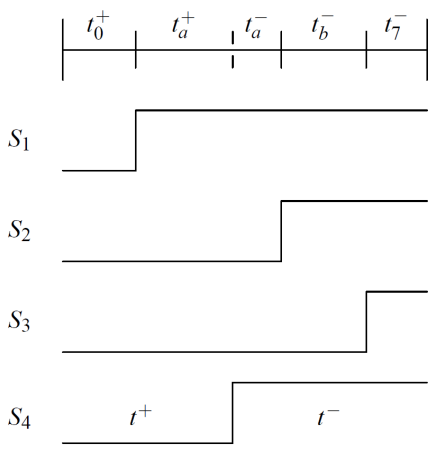

Fig. 4. Tiempos de conmutación.

$$
\begin{gathered}
t^{+}=t_{+0}+t_{+a} \\
t^{-}=t_{-a}+t_{-b}+t_{-7}
\end{gathered}
$$


$\mathrm{Al}$ sustituir cada una de las expresiones definidas anteriormente en (8), se obtiene la expresión de la tensión homopolar en forma reducida (12).

$$
V_{0}=\bar{V}\left[\left(t_{b}-t_{a}\right)+3\left(t_{7}-t_{0}\right)\right]+\Delta V\left(t^{+}-t^{-}\right)
$$

Esta tensión homopolar es función de los tiempos de modos activos y pueden ser calculados de la misma forma como se realiza en un inversor trifásico convencional. De la misma forma, la tensión homopolar es función de los modos inactivos y los tiempos de conmutación de la cuarta rama. La cuarta rama muestra un componente adicional de tensión homopolar, el cual puede ser distribuido de forma arbitraria para cumplir los requerimientos de tensión homopolar.

Al utilizar los tiempos residuales (tiempos que no gastan los modos activos) y los tiempos de conmutación de la cuarta rama del inversor en una forma adecuada, se puede derivar las expresiones de máxima y mínima tensión homopolar como se muestra en la siguiente sección.

\section{CÁLCULO DEL VALOR MÁXIMO Y MÍNIMO DE TENSIÓN HOMOPOLAR}

Para obtener el valor máximo y mínimo de tensión homopolar $V_{0}$ en función de los tiempos de conmutación del inversor, se recurre a (12) y substituyendo los valores numéricos medios e incrementales se obtiene la expresión analítica de la tensión homopolar con ocho interruptores valida en un sector comprendido entre dos modos activos $\mathrm{y}$ adyacentes.

$V_{0}=\frac{V_{c}}{2 \sqrt{3}}\left(t_{b}-t_{a}\right)+\frac{\sqrt{3} V_{c}}{2}\left(t_{7}-t_{0}\right)+\frac{\sqrt{3} V_{c}}{2}\left(t^{+}-t^{-}\right)$

Se define un vector giratorio de tensión $v_{d q}$ constante como la tensión del inversor a ser suministrada por el inversor y se asumiendo que se encuentra en el subespacio del sector dado, se tiene:

$$
\begin{array}{ll}
v_{d}=v_{d q} \cos \alpha & (\alpha=\angle a, \ldots, \angle b) \\
v_{q}=v_{d q} \sin \alpha & (\alpha=\angle a, \ldots, \angle b)
\end{array}
$$

Donde $v_{d q}$ es la magnitud del vector de tensión normalizado y $\alpha$ el ángulo comprendido en el subespacio de dos modos adyacentes ( $a$ y $b$ ).
Sustituyendo (14) y (15) en (7) se obtienen los tiempos de modos activos de conmutación $t_{a}$ y $t_{b}$ en función del valor del vector de tensión $d q$ del inversor dado como sigue:

$$
\begin{gathered}
t_{a}=\frac{\sqrt{2}}{2} v_{d q}(\sqrt{3} \cos \alpha-\sin \alpha) \\
t_{b}=\sqrt{2} v_{d q} \sin \alpha
\end{gathered}
$$

La expresión de tensión homopolar dada por (13) muestra que se puede obtener un máximo o un mínimo de tensión homopolar si se dedica el tiempo residual en el uso de un solo modo no activo, ya sea el modo 0 o el modo 7 y si el tiempo de la cuarta rama se hace igual al periodo de conmutación. A continuación, se explican los dos casos límite.

\subsection{Máxima tensión homopolar}

Al dedicar el tiempo residual al modo 7 (el tiempo en 0 se hace cero) y el tiempo de la cuarta rama $t^{+}$se hace igual al tiempo del periodo de conmutación, se obtiene la máxima tensión homopolar que produce un inversor con cuatro ramas de interruptores. El tiempo en el modo 7 en dicho sector resulta en (18) y está determinado al reemplazar (16) y (17) en (3).

$$
t_{7}=1-\frac{\sqrt{2}}{2} v_{d q}(\sqrt{3} \cos \alpha+\sin \alpha)
$$

Sustituyendo (16), (17) y (18) en (13) se obtiene la expresión de máxima tensión homopolar en función de un vector de tensión $v_{d q}$ dado.

$$
v_{0 \max }=\sqrt{3}-\sqrt{2} v_{d q} \cos \alpha \quad(\alpha=\angle a, \ldots, \angle b)
$$

Se encuentra que en el ángulo de $0^{\circ}$ se hace mínima la tensión homopolar máxima y corresponde al valor límite superior de máxima tensión homopolar en régimen permanente que puede suministrar un inversor trifásico con ocho interruptores al aplicar un vector giratorio $v_{d q}$ constante.

El límite máximo de tensión homopolar está dado por (20) y es válida entre el modo activo $a$ y el modo activo $b$.

$$
v_{0 \text { limite }}^{+}=\sqrt{3}-\sqrt{2} v_{d q}
$$




\subsection{Mínima tensión homopolar}

Al dedicar el tiempo residual al modo 0 (el tiempo en 7 se hace cero) y el tiempo de la cuarta rama $t^{-}$se hace igual al tiempo del periodo de conmutación, se obtiene la mínima tensión homopolar que produce un inversor con cuatro ramas de interruptores. El tiempo en el modo 0 resultante en (21) está determinado al sustituir (16) y (17) en (3).

$$
t_{0}=1-\frac{\sqrt{2}}{2} v_{d q}(\sqrt{3} \cos \alpha+\sin \alpha)
$$

Sustituyendo (16), (17) y (21) en (13) se obtiene la expresión de mínima tensión homopolar en función de un vector de tensión $V_{d q}$ dado.

$$
\begin{gathered}
v_{0 \text { min }}=-\sqrt{3}+\frac{\sqrt{2}}{2} v_{d q} \cos (\alpha)+\sqrt{\frac{3}{2}} v_{d q} \sin (\alpha) \\
(\alpha=\angle a, \ldots, \angle b)
\end{gathered}
$$

Se encuentra que en el ángulo de $60^{\circ}$ se hace máxima la tensión homopolar mínima y corresponde al valor límite inferior de mínima tensión homopolar en régimen permanente que puede suministrar un inversor trifásico con ocho interruptores al aplicar un vector giratorio $v_{d q}$ constante. El límite mínimo tensión homopolar está dado por (23) y es válida entre el modo activo $a$ y el modo activo $b$.

$$
v_{0 \text { limite }}^{-}=-\sqrt{3}+\sqrt{2} v_{d q}
$$

\section{CÁlCULO DE LOS TIEMPOS DE CONMUTACIÓN}

Para el cálculo de los tiempos de conmutación se sigue el procedimiento utilizado en el cálculo de los tiempos de conmutación dados en la modulación de ancho de pulso que utiliza el método conocido de vector espacial en dos dimensiones (Prasad et al., 1997a; Prasad et al., 1997b). Estos tiempos son requeridos para realizar físicamente la modulación de ancho de pulso en un inversor con cuatro ramas de interruptores.

La Figura 5 muestra el área factible del vector de tensión a ser suministrada por el inversor. En esta área se proponen la división del área en dos áreas principales. La primera la denotamos como Área 1 y es el lugar en la que se puede suministrar tensión homopolar cuando los tiempos de los modos inactivos $t_{0} \quad$ y $t_{7}$ son iguales. El asumir esta distribución de los tiempos inactivos logra una mayor simetría de las conmutaciones visto en cada periodo de conmutación, con lo cual, genera un menor número de componentes armónicas (Hava et al., 1999; Jacobina et al., 1997). Se calcula el límite para la condición establecida en esta Área 1 en (24) de forma similar al procedimiento empleado en el cálculo del límite de tensión homopolar.

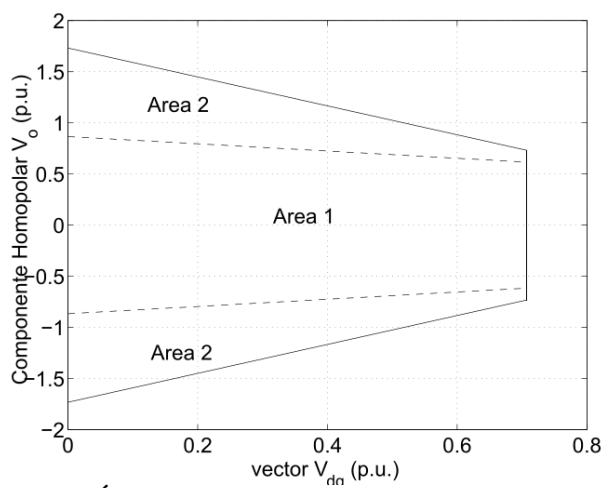

Fig. 5. Áreas factibles del vector del inversor. Límite superior, máxima tensión homopolar. Límite inferior, máxima tensión homopolar $\left(t_{0}=t_{7}\right)$.

$$
v_{0 \text { limite } 1}=\frac{\sqrt{3}}{2}-\frac{\sqrt{3}}{4} v_{d q}
$$

El segundo área, señalada como Área 2, determinada entre el límite del Área 1 y el límite de tensión homopolar total del inversor con cuatro ramas de interruptores. Esta área se hace activa una vez se determina si la tensión homopolar del inversor supera el límite de tensión homopolar del área 1. En esta área se libera la restricción de los modos no activos y se fija el tiempo de de conmutación de la cuarta rama $t^{+}$al valor del tiempo del periodo de conmutación.

Según lo expuesto anteriormente, el cálculo de los tiempos se realiza dependiendo del área en el cual resida el componente homopolar del vector de tensión a ser suministrada por el inversor. $\mathrm{Si}$ el vector de tensión del inversor se encuentra en el Área 1 se aplican (25) a (28) que resumen el cálculo de los tiempos activos e inactivos del inversor con cuatro ramas en dicha área.

$$
\begin{gathered}
\mathbf{t}_{a b}=\mathbf{T}_{a b} \mathbf{v}_{d q} \\
t_{0}=t_{7}=\frac{1-\left(t_{a}+t_{b}\right)}{2} \\
t^{-}=1-t^{+} \\
t^{+}=\frac{v_{0}}{\sqrt{3}}-\frac{\left(t_{b}-t_{a}\right)}{6}-\frac{1}{2}
\end{gathered}
$$


Donde la (25) es la representación simplificada de (7) y $\mathbf{T}_{a b}$ es una matriz depende del sector en el que se encuentre la tensión a ser suministrada por el inversor. De (26) se deduce de (3) y la condición de tiempos de modos activos iguales. (28) se determina a partir de (13) y (27), las cuales determinan el tiempo de conmutación de la cuarta rama.

En el Área 2 el cálculo de los tiempos de los modos activos se realiza de la misma forma como en el Área 1 y los tiempos de los modos inactivos y de la cuarta ramas se definen como sigue:

$$
\begin{aligned}
t_{7} & =\frac{v_{0}}{\sqrt{3}}-\frac{t_{a}}{3}-\frac{2 t_{b}}{3} \\
t_{0} & =1-t_{a}-t_{b}-t_{0} \\
t^{+} & =1 \\
t^{-} & =0
\end{aligned}
$$

Donde el tiempo $t_{7}$ está dado por la (29) y se obtiene de reemplazar las condiciones dadas en el Área 2 y las expresiones (3) y (13). De (30) se deduce de cumplir (3). Los tiempos de conmutación de la cuarta rama se basan en las condiciones del Área 2.

Para la implementación de la modulación es necesario calcular el instante de conmutación de cada rama del inversor teniendo en cuenta los tiempos y el modo (0 ó 7) en el que comienza el periodo. Por lo tanto, se resume como sigue:

$$
\begin{aligned}
\mathbf{t}_{R} & =\mathbf{R}_{0} \mathbf{t}_{0 a b} \\
\mathbf{t}_{R} & =\mathbf{R}_{7} \mathbf{t}_{7 b a}
\end{aligned}
$$

Donde $\quad \mathbf{t}_{0 a b}=\left[\begin{array}{lll}t_{0} & t_{a} & t_{b}\end{array}\right]^{T}, \quad \mathbf{t}_{7 b a}=\left[\begin{array}{lll}t_{7} & t_{b} & t_{a}\end{array}\right]^{T} \quad \mathrm{y}$ $\mathbf{t}_{R}=\left[\begin{array}{llll}t_{R 1} & t_{R 2} & t_{R 3}\end{array}\right]^{T}$. El vector $\mathbf{t}_{R} \quad$ contiene ordenados los instantes en los que tiene que cambiar de estado las ramas 1, 2 y 3 del inversor en un periodo de conmutación. Las matrices $\mathbf{R}_{0} \mathrm{y}$ $\mathbf{R}_{7}$ dependen del sector en el que se encuentre $\mathbf{v}_{d q 0}$, la primera genera $\mathbf{t}_{R}$ para el caso en el que el periodo de conmutación coincida con el modo 0 y la segunda en modo 7. El cálculo de estas matrices es muy sencillo y consiste en combinar convenientemente los tiempos $t_{a}, t_{b}, t_{0}$ y $t_{7}$ para el que el inversor esté en cada modo el tiempo especificado y en el orden adecuado para que las transiciones se produzcan siempre entre modos adyacentes. El tiempo de conmutación de la cuarta rama está determinado por el tiempo $t^{+}$, por tal razón no se incluye en las matrices $\begin{array}{llll}\mathbf{R}_{0} & \text { y } & \mathbf{R}_{7}\end{array}$.
Como ejemplo se muestran los valores de las matrices $\mathbf{T}, \mathbf{R}_{0}$ y $\mathbf{R}_{7}$ para el primer sector como sigue:

$$
\begin{aligned}
\mathbf{T}_{a b}=\sqrt{2}\left[\begin{array}{cc}
\frac{\sqrt{3}}{2} & \frac{-1}{2} \\
0 & 1
\end{array}\right] \\
\mathbf{R}_{0}=\left[\begin{array}{lll}
1 & 0 & 0 \\
1 & 1 & 0 \\
1 & 1 & 1
\end{array}\right] \\
\mathbf{R}_{7}=\left[\begin{array}{lll}
1 & 1 & 1 \\
1 & 1 & 0 \\
1 & 0 & 0
\end{array}\right]
\end{aligned}
$$

\section{SIMULACIÓN Y RESULTADOS EXPERIMENTALES}

En esta sección se describe la implementación de los límites de tensión homopolar siguiendo el análisis obtenido en las secciones anteriores.

Para validar el procedimiento descrito en las secciones anteriores se crea un modelo de simulación en Simulink de Mathworks. En esta simulación se pueden determinar cuatro bloques fundamentalmente. El primer bloque consiste en determinar el valor de máxima tensión homopolar que puede suministrar el inversor y con base en este valor determinar si se sobrepasa o no los límites permitidos.

A continuación se determina el área en la que se encuentra la tensión del inversor y decide que bloque de ecuaciones son requeridas para el cálculo.

Una vez se conoce el área en la que se encuentra el vector de tensión del inversor homopolar, se determinan los tiempos de los modos activos e inactivos y con estos se obtienen los tiempos de conmutación requeridos por cada una de las ramas del inversor de cuatro ramas.

Los bloques anteriores son implementados en simulación y los resultados obtenidos para una tensión especificada de $V_{d}=40 \mathrm{~V}, V_{q}=40 \mathrm{~V}$, $V_{0}=50 \mathrm{~V}$ y tensión en el condensador de $200 \mathrm{~V}$. La figura 6 muestra la salida del inversor después de realizar la transformada de Park a las tensiones de fase dadas en los terminales de salida del inversor. Se observa que los valores de tensión siguen sus referencias en coordenadas $0 d q$. 

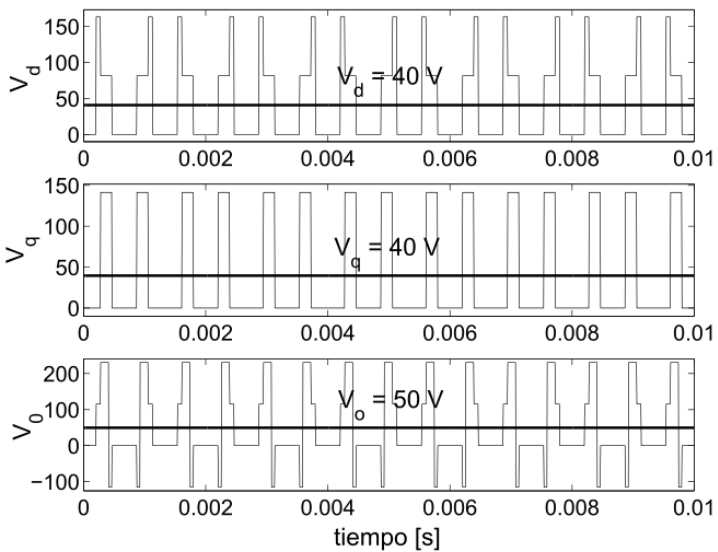

Fig. 6. Resultados simulados en Simulink de la modulación de ancho de pulso utilizando la técnica de vector espacial. Valores promedio de las

tensiones $\left(V_{d}=40 \mathrm{~V}, V_{q}=40 \mathrm{~V}\right.$ y $\left.V_{0}=50 \mathrm{~V}\right)$.

Tensión en el condensador 200 V. Frecuencia de conmutación $2 \mathrm{kHz}$.

Por otra parte, se implementa un prototipo para validar los resultados obtenidos. Este prototipo consta de un inversor trifásico de Skiip 102 GDL 120303 WT de SEMIKRON que opera con una tensión del condensador de $200 \mathrm{~V}$ y sin carga.

El inversor, a su vez, es controlado por medio de una FPGA en la que se implementan los tiempos de conmutación de cada una de las ramas del inversor. Estos tiempos son enviados a la FPGA por medio de un PC en el que corre Simulink con RealTime WorkShop bajo Linux de la empresa MathWorks.

El PC se encarga en determinar el sector y el valor de tiempo a ser asignado en cada rama y enviar dicha información a través de una interface de entradas/salidas a la FPGA.

La tensión de salida del inversor es medida y se aplica la anti transformada de Park para verificar los resultados como se muestra en la Figura 7. Estos datos son adquiridos utilizando un osciloscopio digital DL2700 de la marca Yokogawa de 8 bits y 500MS/s.

La frecuencia de conmutación empleada en el prototipo es de $1.5 \mathrm{Khz}$. Por último, Se observa que los resultados experimentales siguen fielmente los resultados simulados. Este procedimiento permite realizar un control adicional a la tensión homopolar.
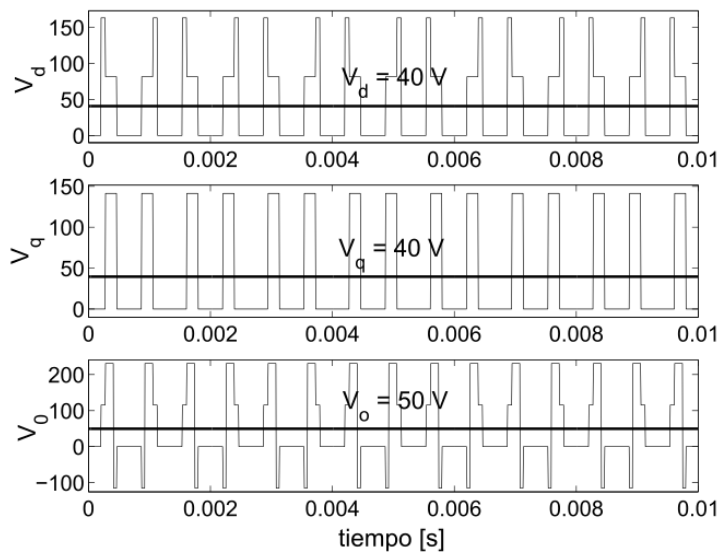

Fig. 7. Resultados experimental de la modulación de ancho de pulso utilizando la técnica de vector espacial. Valores promedio de las tensiones

$$
\left(V_{d}=40 \mathrm{~V}, V_{q}=40 \mathrm{~V} \text { y } V_{0}=50 \mathrm{~V}\right) \text {. }
$$

\section{CONCLUSIONES}

Se obtiene que el cálculo de los tiempos activos no depende de la tensión homopolar a ser suministrada por el inversor, por tal razón es posible ver el inversor de cuatro ramas como una extensión del inversor sin hilo neutro.

Se destacan las expresiones de máximo y mínimo valor de tensión homopolar al igual que las expresiones que suministran los valores límites de tensión. Estos límites facilitan la implementación de algoritmos de control y hacen que estos operen en la región factible del inversor, evitando de esta forma la pérdida de control en regiones no contempladas por los diseños.

Se destaca la capacidad de suministro de tensión homopolar en régimen permanente del inversor de cuatro ramas es mayor que el inversor de tres ramas. Al igual que se observa que un inversor de tres ramas puede trabajar en una región similar a las cuatro ramas si este aumenta en gran medida la tensión de los condensadores.

Se ha propuesto un algoritmo de cálculo de los tiempos inactivos en inversores de cuatro ramas, el cual hace uso de toda la capacidad del inversor, al igual que se minimizan las pérdidas de conmutación con un menor contenido de armónicos. 


\section{REFERENCIAS}

Dai, N.-Y., Wong, M.-C., \& Han, Y.-D. (2006). "Application of a three-level NPC inverter as a three-phase four-wire power quality compensator by generalized 3DSVM". IEEE Transactions on Power Electronics, 21(2), 440-449.

Hava, A. M.; Kerkman, R. J. and Lipo, T. A. (1999). "Simple analytical and graphical methods for carrier-based PWM-VSI drivers", IEEE Transactions on power electronics, vol. 14, pp. 49-61.

Holtz, J. (1994). "Pulsewith modulation for electronic power convertion," Proceeding of the IEEE, vol. 82, pp. 1194-1214.

Jacobina, C. B.; Lima, A. M. N. and Silva, E. R. C. D. (1997). "PWM space vector based in digital scalar modulation," IEEE Power Electronics Specialist Conference (PESC-97), pp. 606-611.

Pinzón A., O. (2015). “Algoritmo para la generación de tensión homopolar en inversores trifásicos con tres ramas de interruptores". Revista Colombiana de Tecnologías de Avanzada, Vol. 1, No. 23.

Prasad, V. H.; Boroyevich, D. and Zhang, R. (1997). "Analysis and comparison of space vector modulation schemes for a four-leg voltage source inverter," Virginia Power Electronic Center, VPEC, pp. 864-871.

Prasad, V. H.; Boroyevich, V. H. and Zhang, R. (1997). "Comparison of high frequency PWM algorithms for voltage source inverters," Virginia Power Electronic Center, VPEC, pp. 115-122, 1997.
Shen, D. and Lehn, P. W. (2002) "Fixed-frecuency space-vector modulation control for trheephase four-leg active power filters", IEE Proceeding Electronic Power Application, Vol. 149, No. 4, pp. 268-274.

Srikanthan, S., and Mishra, M. K. (2010). "Modeling of a four-leg inverter based DSTATCOM for load compensation". En 2010 International Conference on Power System Technology (POWERCON) (pp. 1-6).

Xiao-pingYang, Zhang, Y., \& Zhong, Y. (2008). "Three-phase four-wire DSTATCOM based on a three-dimensional PWM algorithm". En Third International Conference on Electric Utility Deregulation and Restructuring and Power Technologies, 2008. DRPT 2008 (pp. 2061-2066).

Zhang, R.; Prasad, V. H. and Boroyevich, D. (2002) "Three-dimensional space vector modulation for four-leg voltage-source converters," IEEE Transactions on Power Electronics, vol. 17, no. 3, pp. 314-326.

Zhang, M., Atkinson, D., and Armstrong, M. (2012). "A zero-sequence component injected PWM method with reduced switching losses and suppressed common-mode voltage for a three-phase four-leg voltage source inverter". En IECON 2012 - 38th Annual Conference on IEEE Industrial Electronics Society (pp. 5068-5073). 